\title{
Wireless Remote Data Transmission Technology be Used in the Grating Demodulation Instrument ${ }^{*}$
}

\author{
Meng Xian-wei, Yang Zhuo-jing, Zhang Qing, Shi Yan-xin, Zhang Xiao-fei \\ Centre for Hydrogeology and Environmental Geology Survey, CGS, Key Laboratory of Geological Environment Monitoring \\ Technology, MLR, Baoding, 071051, China
}

\begin{abstract}
According to the property of GPRS network transmission, wireless remote data transmission technology is applied to the monitoring data of fiber Bragg grating, through the GPRS network to transmit the FBG monitoring data to data monitoring center, in the numerical control center can understand the monitoring site information, in order to achieve real-time monitoring of timing, automatic, unattended. In this paper we introduced the system structure design of the FBG monitoring system, We discussed the software of the wireless remote data transmission and the data pretreatment. As a result, it is suited that use GPRS wireless communication mode to realize the grating remote transmission of monitoring information is feasible.

Index Terms - GPRS network, Wireless Remote Data Transmission, FBG monitoring system.
\end{abstract}

\section{Introduction}

GPRS (General Packet Radio Service) is based on GSM network, and to provide users with the network service of high-speed packet data. In recent years, with the continuous optimization of GPRS network, network quality is enhanced unceasingly, GPRS is increasingly used in various industries. GPRS has many advantages, such as: high resource utilization, large network coverage, network access speed, high transmission speed and support a variety of transport protocols, etc. Compared with traditional railway network, it has incomparable advantages, it can completely meet the needs of the fiber Bragg grating remote long-distance monitoring. Therefore, it is great significance that wireless remote data transmission technology is applied to the grating monitor to realize automation for the monitoring and control of fiber Bragg grating ${ }^{[1-2]}$.

\section{System Design}

FBG monitoring system consists of fiber Bragg grating sensor array, the grating demodulation instrument, data monitoring center. The wavelength data of the fiber Bragg grating sensors is collected by the grating monitor, the collecting data is saved and packed by demodulation instrument, through wireless data transmission module the collecting data is sent to the GPRS network, and be received by the server computer that is set the IP address in advance. System structure is shown in figure $1^{[3]}$.

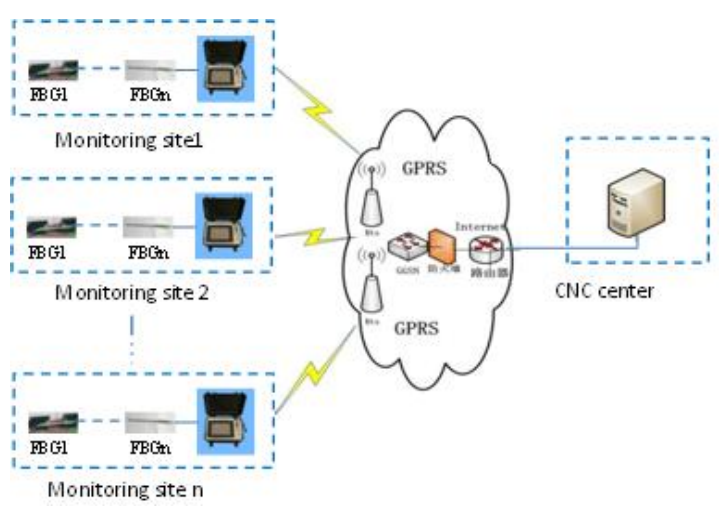

Fig.1 Diagram of fiber Bragg grating monitoring system

\section{Hardware Design}

In the grating demodulation instrument, the microcontroller processor is used to implement the sensor data collection, preservation, packaging. Through a serial port, the packaging data is sent to the wireless transmission module. The wireless data transmission module is used that it is built TCP/IP protocol GPRS. The module connects with the microprocessor through the serial port. Before use, all the parameters are set, includes the module ID number, communication baud rate, its way of working, remote communication data center address and port number, etc. After the parameters are set, it is stored inside the module of FALSH $^{[4]}$.

Setup has completed, exit the settings mode into data transmission mode. When the communications module loading power, first read the internal configuration parameters, automatically enter the transfer mode has been set, and then $\log$ in to the GPRS network, link building and monitoring center, and always keep a link. By the serial port, the selecting data is sent to the transmission module, and packaged based on the TCP/IP protocol, and then be sent to the GPRS network.

The biggest advantage is that if the communication is not online, will automatically redial, and keep the link has been opened, it has the very high real-time performance. At the same time, operating instructions and information exchange is done inside the module automatically.

\footnotetext{
* His work is supported by the Geological Survey Project of CGS(1212011140020).
} 


\section{Software Design}

Wireless remote data transmission software is written in labview software platform. Software functions are data acquisition, data processing, data, etc. The software adopts the design of the modular, it can improve the flexibility of software, easy software function increase and adjust later.

\section{A. data collection}

The wavelength and power of the data be read by the FBG monitoring system from the demodulation module, and the measurement of physical parameters be counted according the parameters of the sensor, the wavelength and test results is saved in the file that named by test time ${ }^{[5]}$. Data acquisition software implementation process chart is shown in figure 2 .

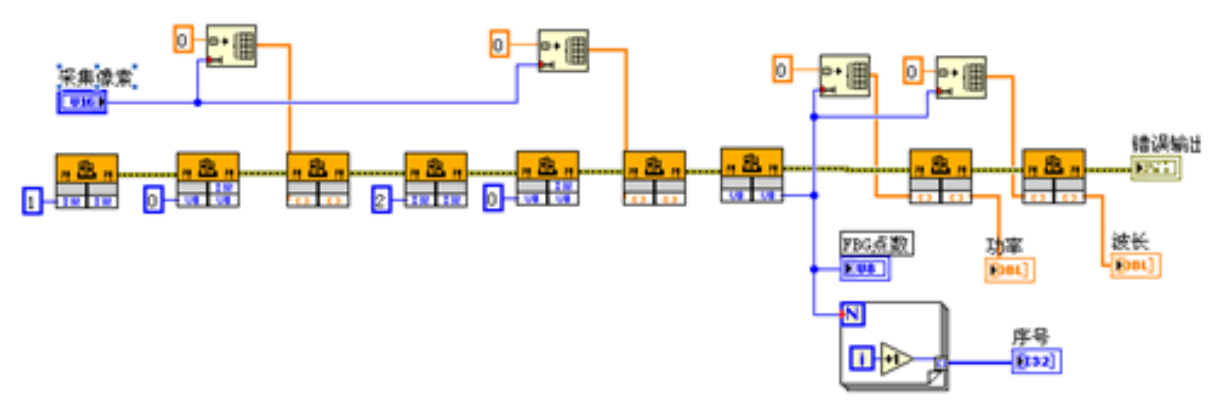

Fig.2 Data acquisition software implementation chart

\section{B. Pretreatment of data transmission}

In the process of wireless transmission, there will be a loss of data and sends the wrong situation. Therefore, in order to ensure the data accurate transmission, add check code to transmit data as a signature, it transmitted to the receiver together with the measured data, the receiver according to the received data to recalculate the check code, if the check code is different with the check code from the sender, indicates that data is error during transmission.
The most common ways of check are xor, calibration and cyclic redundancy check, in this software, the checksum method be used to calculate the check code. The calculation method is: Every 8 bytes of the transmit data be added, lost to carry; invert the calculation results, then the results add 1 , the result is the check code. Will be finished all the bytes in the data transmission, all the check calculated code be connected together, this is the check code to transmit data. Check code calculation software flow chart is shown in figure 3 .

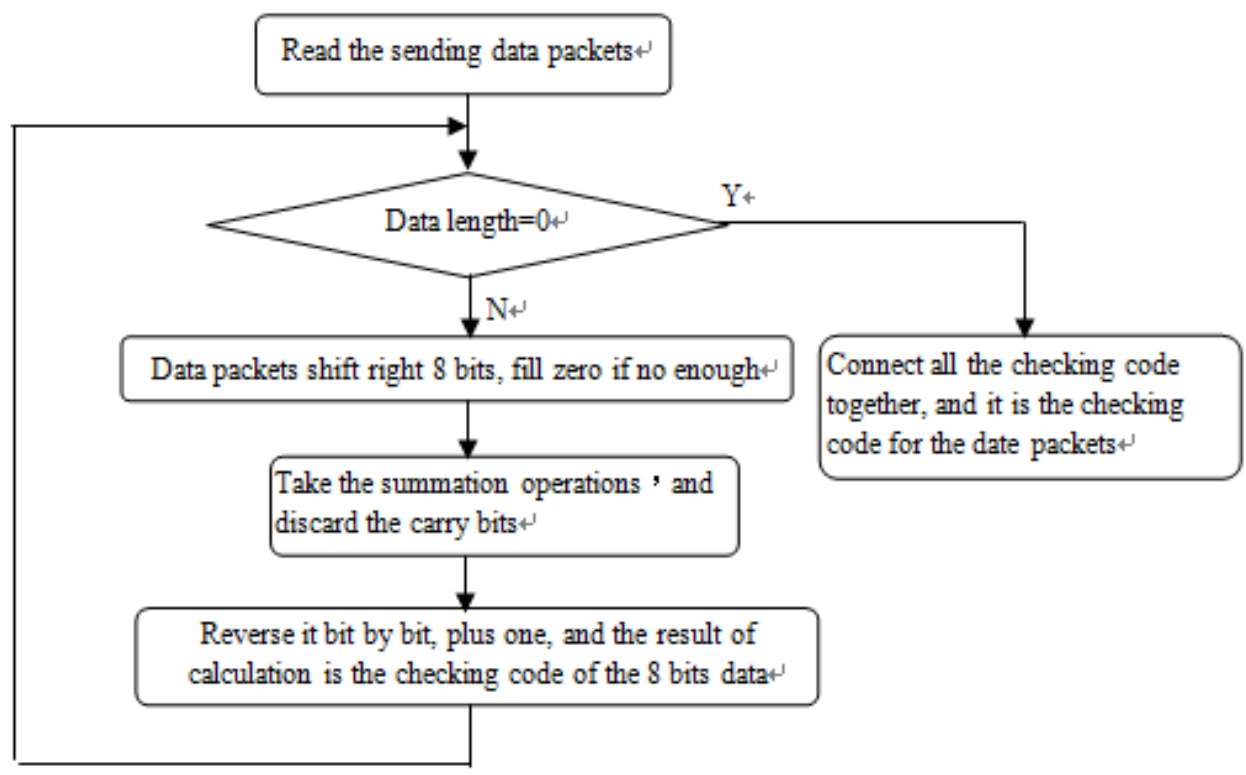

Fig. 3 Checksum calculation flow chart

\section{Delivery of the monitoring data}

The master-slave question-and-answer method of communication be used in FBG monitoring system and the control center, when the data acquisition is completed, the instrument to send data transmission request instructions to control center, the control center receiving the order, and to send response signal, the response signal be received by the 
instrument, the wavelength of fiber grating sensor data and the result data be sent to the center, and send the end signal, the data transmission is completed. Monitoring data sending software flow chart is shown in figure 4.

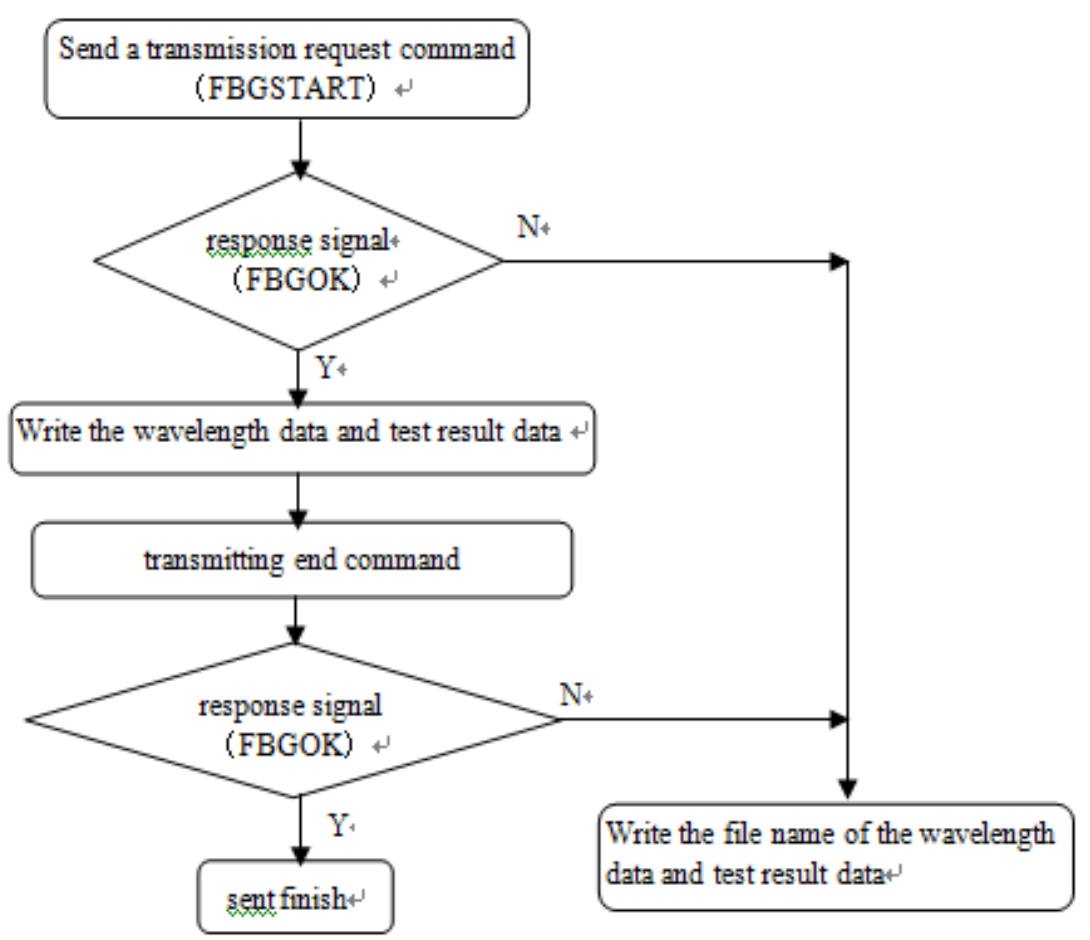

Fig 4 Monitoring data sending flow chart

\section{Receiving Software Design}

The control center is consisted of by wireless remote data receiving software and database. The server of control center has a fixed IP address, and using this IP address to access GPRS network. The server can receive the data that send from each the GPRS wireless communication module setting IP address, these data are analyzed and the processed, storage, fault diagnosis. The receiving software interface of wireless remote transmission is shown in figure 5.

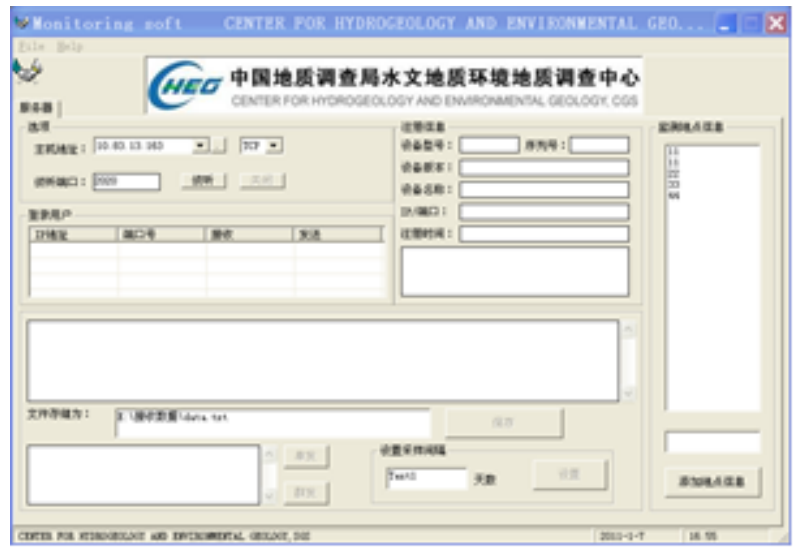

Fig.5 The receiving software interface of wireless remote transmission

\section{Conclusion}

FBG monitoring system can be monitoring of multiple distributed fiber grating monitoring stations at the same time, and to ensure the timeliness, accuracy of data acquisition and data transmission reliability. This system uses the GPRS wireless communication mode to realize the grating remote transmission of monitoring information, this way has the advantages of the building maintenance costs low, wide coverage, strong anti-jamming capability, it can understand the monitoring scene information in the control center, thus has broad application prospects.

\section{References}

[1] Meng Xianwei, Zhang Qing, Shi Yanxin, Han Yongwen, Jiang Fan. Development of Wavelength Demodulation for Fiber Bragg Grating. journal of jilin university raeth science edition, 2009, 39(2): 342 346.

[2] Zhang Qing, Shi Yanxin, Meng Xianwei, Experimental Research of Landslide Monitoring Based on FBG, Chinese Geophysical Society, Chinese geophysical 24th annual meeting. Beijing: China Land Press, 2008: 451.

[3] Zhao Yong. Principles and application technology of optical fiber sensors. Beijing: tsinghua unverdity press, 2007: 129 134.

[4] Wang Yonghong, Xu Wei, Hao Liping, ARM Cortex-M3 Microprocessor Principle and Practice, Beijing university of aeronautics and astronautics press, 2008: 22 50. 